\title{
Sensor-based gait analyses of the six-minute walk test identify qualitative improvement in gait parameters of people with multiple sclerosis after rehabilitation
}

\author{
Pål Berg-Hansen ${ }^{1} \mathbb{D}$. Stine Marit Moen ${ }^{2} \cdot$ Andreas Austeng $^{3} \cdot$ Victor Gonzales ${ }^{4}$. Thomas Dahl Klyve ${ }^{2}$. \\ Henrik Negård ${ }^{2} \cdot$ Trine Margrethe Seeberg $^{4} \cdot$ Elisabeth Gulowsen Celius ${ }^{1,5} \cdot$ Frédéric Meyer $^{3}$
}

Received: 16 September 2021 / Revised: 23 January 2022 / Accepted: 24 January 2022 / Published online: 15 February 2022

(c) The Author(s) 2022

\begin{abstract}
The aim of this work was to determine whether wearable inertial measurement units (IMUs) could detect gait improvements across different disability groups of people with Multiple Sclerosis (pwMS) by the six-minute walk test (6MWT) during a rehabilitation stay in a specialized rehabilitation center. Forty-six pwMS and 20 healthy controls (HC) were included in the study. They performed the 6MWT with two inertial measurement units (IMUs) placed on the feet. Thirty-two of the pwMS were retested at the end of the stay. PwMS were divided in a mild-disability and a moderate-disability group. The 6MWT was divided in six sections of 1 min each for technical analysis, and linear mixed models were used for statistical analyses. The comparison between the two disability groups and $\mathrm{HC}$ highlighted significant differences for each gait parameter (all $p<0.001)$. The crossing effect between the test-retest and the two disability groups showed greater improvement for the moderate-disability group. Finally, the gait parameter with the higher effect size, allowing the best differentiation between the disability groups, was the foot flat ratio $\left(R^{2}=0.53\right)$. Gait analyses from wearable sensors identified different evolutions of gait patterns during the 6MWT in pwMS with different physical disability. The measured effect of a short-time rehabilitation on gait with 6MWT was higher for pwMS with higher degree of disability. Using IMUs in a clinical setting allowed to identify significant changes in inter-stride gait patterns. Wearable sensors and key parameters have the potential as useful clinical tools for focusing on gait in pwMS.
\end{abstract}

Keywords Multiple sclerosis $\cdot$ Six-minute walk $\cdot$ Gait analysis $\cdot$ Inertial measurement units $\cdot$ Wearable sensors . Biomechanics

Pål Berg-Hansen and Stine Marit Moen have contributed equally to this work.

Pål Berg-Hansen

pal.berg-hansen@ous-research.no

1 Department of Neurology, Oslo University Hospital, Ullevål, Sognsvannsveien 20, 0372 Oslo, Norway

2 MS Center Hakadal, Hakadal, Norway

3 Department of Informatics, University of Oslo, Oslo, Norway

4 SINTEF Digital, Smart Sensor Systems, Oslo, Norway

5 Institute of Clinical Medicine, University of Oslo, Oslo, Norway

\section{Introduction}

Multiple sclerosis (MS) is a chronic disease with onset in young adulthood and characterized by inflammation and neurodegeneration [1]. Walking disability is one of the most common symptoms in people with MS (pwMS) with major impact on physical function and quality of life [2]. The Expanded Disability Status Scale (EDSS) [3] is the most used measure of disability in MS. The scale ranges from 0 (no disability) to 10 (death), heavily depending on ambulation in the higher range and with several limitations [4]. In addition, clinicians use walking tests, both performance-based and patient-reported outcome measures, to assess gait function and the impact of MS on walking. The six-minute walk test (6MWT) [5] is commonly applied to measure endurance walking capacity and has proven to relate better to daily life walking than shorter tests. The 
6MWT has been found to detect clinically meaningful improvement after physical rehabilitation [6]. The tests that are used in today's clinical practice are in general not sensitive enough to detect gait problems and changes in gait function among pwMS with low physical disability, in contrast to the gait measurements and analysis possible in specialized laboratories and research settings [7]. Several studies using automated gait analyses have been published as reviewed by Shanahan et al. [7] and by Brichetto et al. [8]. As an example, it has been shown a greater effect of physical rehabilitation among pwMS with slower gait speed at baseline [9]. Mobility assessment is important in the clinical management of pwMS. Portable technologies with ambulatory monitoring systems and wearable sensors are a rapidly evolving field [10-13]. Sensors can be positioned on different parts of the body depending on the situation. An IMU placed on the waist is probably the most adapted to real-life monitoring [14], while having IMUs placed on the shins or on the foot provide more detailed parameters in a clinical situation $[15,16]$. Today inertial measurement units (IMUs) are frequently used in research studies for assessment of movement pattern and stability of persons with gait limitations like elderly that have an increased risk of falling, persons with Parkinson's disease, epilepsy, stroke, and MS [8, 17]. However, wearable unobtrusive sensors may also be useful in clinical settings for example to improve diagnostics, objectively test the effect of interventions and in monitoring disease development. Previous studies have shown that pwMS with moderate or severe walking disability had reduced gait speed, shorter stride length and prolonged swing phase, double limb support time, and stride time, compared to healthy controls (HC) [18]. It has also been shown a greater step time variability in pwMS compared to HC [19]. However, there are fewer studies on what characterizes gait in pwMS with mild disability. For example, there has been identified reduced speed, stride length, and double limb support in pwMS with mild disability compared to healthy controls $[20,21]$. However, the findings are not consistent, probably due to lack of statistical power and different study populations. A few other studies have utilized body-worn sensors on the $6 \mathrm{MWT}$ in pwMS. In a recent review, 5 of 28 studies were on pwMS [22]. Among pwMS with different disability levels, it has been shown that different parameters show clinically meaningful change after rehabilitation [23], and instrumented gait analyses have provided a plethora of metrics for quantifying concurrent factors contributing to gait deterioration and for determining change and responsiveness of interventions [7]. In the present study, we have examined pwMS together with healthy controls (HC) using body-worn IMUs during the 6MWT. The primary aim was to determine whether IMUs could be used to detect improvement in gait parameters across different disability groups during a conventional rehabilitation stay in an MS rehabilitation unit. It was hypothesized that the parameters obtained using wearable sensors would allow for a more sensitive assessment of the pwMS than the standard analysis. We further wanted to investigate the walking impairment in relation to standard walking tests across different disability levels and to explore the potential of IMU-generated parameters as a clinical monitoring tool to detect relevant changes during rehabilitation.

\section{Methods}

\section{Participants}

Several pilots were done in advance to gain information on the number of subjects needed and adjust practical setup. PwMS who were admitted to a rehabilitation stay with focus on any issue that could influence physical function and gait were asked to participate. The participants were allowed to use their own orthoses or other gait aids and had to be able to walk at least $100 \mathrm{~m}$ with one cane (EDSS $\leq 6)$. All that met the inclusion criteria during the study period were asked to participate in the study. Forty-six pwMS aged 26-67 years (mean 50.2) consented and participated (100\%). Six of the pwMS had primary progressive MS and 40 had relapsing remitting MS. Thirty-two of the patients were tested twice, at the beginning and end of the rehabilitation stay (mean 15, range 7-22 days). Of these, 26 pwMS were able to walk with no aids, three with one orthosis, and three with canes. The pwMS were divided in a mild-disability group $($ EDSS $<4)$ and a moderate-disability group (EDSS $\geq 4$ ). None of the pwMS had changed disease modifying treatment or had any clinical relapses in the last month prior to inclusion. Twelve of the patients were using fampridine which is known to improve walking range in pwMS [24], and of these patients, 10 were retested. One of the participants initiated the drug during the rehabilitation stay and two patients discontinued fampridine. Twenty $\mathrm{HC}$ were recruited among employees at the rehabilitation center and were tested once with the same tests protocol as the pwMS. Clinical data for the pwMS, i.e., time of diagnosis and history of immunomodulatory treatment, were retrieved from the medical files.

\section{Test procedure}

All testings were performed in a dedicated test room with a stable temperature between 22 and $23{ }^{\circ} \mathrm{C}$. The hallway was $30 \mathrm{~m}$ in length with flat concrete underlay in line with the standard test protocol for the 6MWT [25]. All participants signed the written consent form at inclusion. A neurological 
exam was performed by a trained neurologist at the beginning of each stay with assessment of EDSS. Prior to the 6MWT, the patients performed four other clinical tests: The time up and go test [26], single leg stand test [27], timed 25 -foot walk test [28], and the six spot step test [29]. For the 6MWT, the participants were instructed to walk as far as possible in 6 min along the hallway and turn at each end. They were allowed to take brakes during the test. During the test, they were informed at 3 min that they were halfway through the test, and at $5 \mathrm{~min}$ that they had $1 \mathrm{~min}$ left.

\section{Equipment}

Two IMUs (Physiolog $5^{\circledR}$ from GaitUp SA, Lausanne, Switzerland) containing a 3D accelerometer and a 3D gyroscope were placed centered on the dorsum of each foot with an elastic band (Fig. 1). The sampling rate of the accelerometer and gyroscope was set to $128 \mathrm{~Hz}$ with a range of $\pm 8 \mathrm{~g}$ for the accelerometer and $\pm 1000 \%$ for the gyroscope. Fifteen standard gait parameters (i.e., cycle duration, cadence, stance, swing, load ratio, foot flat ratio, push ratio, double support time, stride length, speed, peak swing, foot pitch

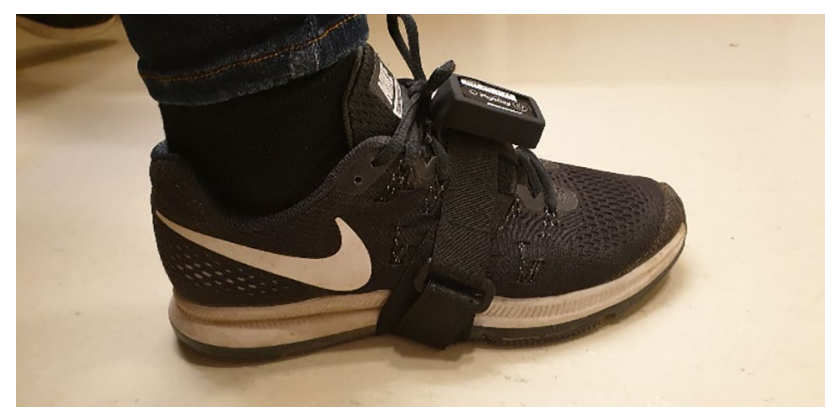

Fig. 1 Positioning of the inertial measurement units angle at heal strike, foot pitch angle at toe off, swing width, and path length) were extracted from the IMU data on the feet using a specialized software for gait analysis (GaitUp Lab $^{\circledR}$, GaitUp SA, Lausanne, Switzerland). The description of the parameters can be found in Table 1. The algorithms used to determine these gait parameters have been validated in different publications on healthy [30], elderly [16], and Parkinson [31] population. The sensors were automatically calibrated by the software using an initial static period, as well as the direction of the first steps. The period of each step when the foot is flat on the ground was used to recalibrate the sensor orientation for each cycle [16]. During the analysis, the two steps before and after each turn, as well as the periods of rest, were removed by the software. The movement of each participant was captured with two cameras for back-up. One GoPro Hero $7^{\circledR}$ camera was mounted on the chest with a GoPro Chesty ${ }^{\circledR}$ strap and directed toward the feet. One GoPro Hero $7^{\circledR}$ camera was mounted on a tripod directed slightly downwards avoiding the face of the test person to be shown on the film.

\section{Data transfer and storage}

Prior to data analyses, data and videos were transferred for safe storage at the Service for sensitive data server. All data handling was unidentified and code key stored separately on an encrypted storage device.

\section{Statistical analysis}

The 6MWT was divided in six sections of 1 min each for technical analysis. To test for normality, a Q-Q plot was used for graphical interpretation and Shapiro-Wilk test was used to test for normality due to small sample sizes. Normally distributed continuous variables were compared using

Table 1 Description of the different parameters determined using the inertial measurement units attached to the feet

\begin{tabular}{|c|c|c|c|c|c|c|}
\hline & Parameter & Definition & Parameter & Definition & Parameter & Definition \\
\hline & $\begin{array}{l}\text { cycle duration } \\
\text { [s] }\end{array}$ & $\begin{array}{l}\text { Time between one foot } \\
\text { strike and the subsequent } \\
\text { foot strike of the same } \\
\text { foot }\end{array}$ & $\begin{array}{l}\text { max angular } \\
\text { velocity } \\
{[\mathrm{deg} / \mathrm{s}]}\end{array}$ & $\begin{array}{l}\text { Maximal angular velocity of } \\
\text { the leg during the swing } \\
\text { phase }\end{array}$ & $\begin{array}{l}\text { stance phase } \\
\text { [\% of cycle } \\
\text { duration] }\end{array}$ & $\begin{array}{l}\text { The entire time that a foot is on the } \\
\text { ground }\end{array}$ \\
\hline & $\begin{array}{l}\text { cadence } \\
\text { [steps } / \mathrm{min}]\end{array}$ & $\begin{array}{l}\text { Number of steps } \\
\text { performed in one minute }\end{array}$ & $\begin{array}{l}\text { foot pitch } \\
\text { angle at heel } \\
\text { strike [deg] }\end{array}$ & $\begin{array}{l}\text { Angle between the foot and } \\
\text { the ground when the heel hit } \\
\text { the ground }\end{array}$ & $\begin{array}{l}\text { swing time } \\
\text { [\% of cycle } \\
\text { duration] }\end{array}$ & $\begin{array}{l}\text { The entire time that a foot is in the } \\
\text { air }\end{array}$ \\
\hline & $\begin{array}{l}\text { stride length } \\
{[\mathrm{m}]}\end{array}$ & $\begin{array}{l}\text { Distance between } \\
\text { successive points of } \\
\text { initial contact of the } \\
\text { same foot }\end{array}$ & $\begin{array}{l}\text { foot pitch } \\
\text { angle at toe- } \\
\text { off }[\mathrm{deg}]\end{array}$ & $\begin{array}{l}\text { Angle between the foot and } \\
\text { the ground when the toe is } \\
\text { released from the ground }\end{array}$ & $\begin{array}{l}\text { load ratio } \\
{[\% \text { of stance }} \\
\text { phase }]\end{array}$ & $\begin{array}{l}\text { Start at the first heel contact of the } \\
\text { lead leg and end at the first contact } \\
\text { of the first metatarsal head of the } \\
\text { lead leg }\end{array}$ \\
\hline & speed $[\mathrm{m} / \mathrm{s}]$ & $\begin{array}{l}\text { Distance travelled per } \\
\text { unit of time }\end{array}$ & $\begin{array}{l}\text { swing width } \\
{[\% \text { of stride }} \\
\text { length] }\end{array}$ & $\begin{array}{l}\text { Ratio between the lateral } \\
\text { displacement of the foot } \\
\text { during the swing and the } \\
\text { stride length }\end{array}$ & $\begin{array}{l}\text { foot flat ratio } \\
{[\% \text { of stance }} \\
\text { phase }]\end{array}$ & $\begin{array}{l}\text { Start at the first contact of the first } \\
\text { metatarsal head of the lead leg and } \\
\text { end at the heel off of the lead leg }\end{array}$ \\
\hline 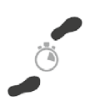 & $\begin{array}{l}\text { double contact } \\
{[\% \text { of cycle }} \\
\text { duration] }\end{array}$ & $\begin{array}{l}\text { Time spent with both } \\
\text { feet on the ground during } \\
\text { one gait cycle }\end{array}$ & $\begin{array}{l}\text { path length } \\
{[\% \text { of stride }} \\
\text { length] }\end{array}$ & $\begin{array}{l}\text { 3D distance travelled by the } \\
\text { foot during the swing } \\
\text { compared to the stride } \\
\text { length }\end{array}$ & $\begin{array}{l}\text { push ratio } \\
{[\% \text { of stance }} \\
\text { phase }]\end{array}$ & $\begin{array}{l}\text { Start at the heel off of the lead leg } \\
\text { and end at the toe off of the lead leg }\end{array}$ \\
\hline
\end{tabular}


independent samples t test. Mann-Whitney $U$ test was used to compare ordinal variables, and Pearson's Chi-square test was used to compare dichotomous variables. Paired samples $t$ test was used to compare repeated measures. Linear mixed models were used to compare differences in walking parameters (e.g., speed, ground contact time, cadence, loading ratio, and foot flat ratio). The variability was calculated as the difference between the left and the right legs over the whole 6MWT. All the steps included in the global analysis are also included in the variability analysis. Disability group, test-retest, and section were defined as fixed effect and intercept on subject as random effect. Marginal $R^{2}$ and conditional $R^{2}$ were presented to assess, respectively, fixed and random effect size. Values over 0.02 were considered small, 0.15 medium, and 0.35 large effect sizes [32]. A significance level was set as $p<0.05$, and a Bonferroni pairwise comparison post hoc test was then applied to identify differences between sections and disability groups. The Bonferroni correction was applied to the $p$ value obtained, so the $\mathrm{p}$ value is corrected, and the alpha level stays as determined (0.05). The statistical comparisons were obtained using Jamovi Software (Jamovi project 2020, Version 1.2).

\section{Results}

The demographics and clinical data of pwMS and HC are given in Table 2. The total distance walked during the 6MWT was significantly longer for the $\mathrm{HC}$ group (mean 694, SD $90 \mathrm{~m}$ ) compared to the mild-disability group (540, $88 \mathrm{~m}$ ), who walked longer than the moderate-disability group $(421,99 \mathrm{~m})($ all $p<0.001)$. After retesting at the end of the rehabilitation stay, the pwMS improved their walking distance by a mean of $15.9 \mathrm{~m}(p<0.037)$. The moderatedisability group was able to improve the walking distance by a mean of $24.4 \mathrm{~m}$ (SD 38.7, $p=0.016$ ) in contrast to $5.1 \mathrm{~m}$ (SD 43.4, $p=0.67$ ) for the mild-disability group. Test results for the different IMU parameters from the left and right leg averaged for 32 pwMS and $20 \mathrm{HC}$, were analyzed with the mixed model, and are shown in Table 3. There was a significant difference for all parameters from test to retest, except for load ratio and swing width. For foot pitch angle at heal strike, there was a trend toward significance. Most parameters had medium-to-large effect sizes. The foot flat ratio (percentage of the stance phase with the foot flat) had the highest effect size of 0.53 .

There were significant different evolutions of cross-correlation for the parameters cadence, speed, load ratio, and foot flat ratio between the three groups from minute 1 to minute 6 during the test (Fig. 2). The HC group increased cadence, the mild-disability group was stable, and the moderate-disability group decreased cadence during the test. Speed was stable in the $\mathrm{HC}$ group and the mildly disabled group but decreased in the moderately disabled group. Load ratio and foot flat ratio were stable in the HC group, whereas for both pwMS groups load ratio decreased, and in accordance with this, foot flat ratio increased during the test. The parameters with non-significant cross-correlation are presented in supplementary material (Figure S1).

Figure 3 illustrates the baseline IMU parameters for all participants, and results at retest for the two disability groups. For the stance phase, swing phase, double stance, stride length, max angular velocity, toe-off pitch angle, swing width and path length, the moderate-disability group had a better improvement after rehabilitation than the milddisability group. The parameters with non-significant crosscorrelation are presented in supplementary material (Figure S2).

Variability in steps between left and right leg decreased from baseline test to retest, and was significant for the following parameters: stance phase, load ratio, foot flat ratio, push ratio, stride length, gait speed, maximum angular velocity, and path length (Table 4). The correlation coefficients were lower compared to the coefficients obtained for the mean values presented in Table 2, with only small- or medium-effect sizes for all parameters.

Table 2 Demographic and clinical data of healthy controls and people with multiple sclerosis

\begin{tabular}{|c|c|c|c|c|c|c|}
\hline & $\begin{array}{l}\text { Healthy } \\
\text { controls } \\
(n=20)\end{array}$ & PwMS All $(n=46)$ & $\begin{array}{l}\mathrm{p} \text { Value (All pwMS } \\
\text { vs healthy controls) }\end{array}$ & $\begin{array}{l}\text { PwMS mild } \\
\text { disability } \\
(n=20)\end{array}$ & $\begin{array}{l}\text { PwMS moderate } \\
\text { disability }(n=26)\end{array}$ & $\begin{array}{l}p \text { Value (mild vs } \\
\text { moderate-disability } \\
\text { pwMS) }\end{array}$ \\
\hline Age in years, mean (SD) & $47.7(12.3)$ & $50.2(8.3)$ & 0.32 & $49.4(10.3)$ & $50.9(6.5)$ & 0.55 \\
\hline Female/male ( $\%$ female) & $16 / 4(80)$ & $29 / 17(63)$ & 0.25 & $15 / 5(75)$ & $14 / 12(53)$ & 0.14 \\
\hline BMI, mean (SD) & $25.4(3.4)$ & $26.9(4.4)$ & 0.18 & $29.1(3.3)$ & $25.1(4.4)$ & 0.002 \\
\hline EDSS, median (range) & & $4.0(1-6)$ & & $2.8(1-3.5)$ & $4.3(4-6)$ & $<0.001$ \\
\hline $\begin{array}{l}\text { Years since diagnosis, } \\
\text { median (range) }\end{array}$ & & $10.5(0-30)$ & & $9.0(0-30)$ & $11(0-21)$ & 0.77 \\
\hline
\end{tabular}

PwMS People with Multiple Sclerosis, BMI Body Mass Index, SD Standard Deviation, EDSS Expanded Disability Status Scale 


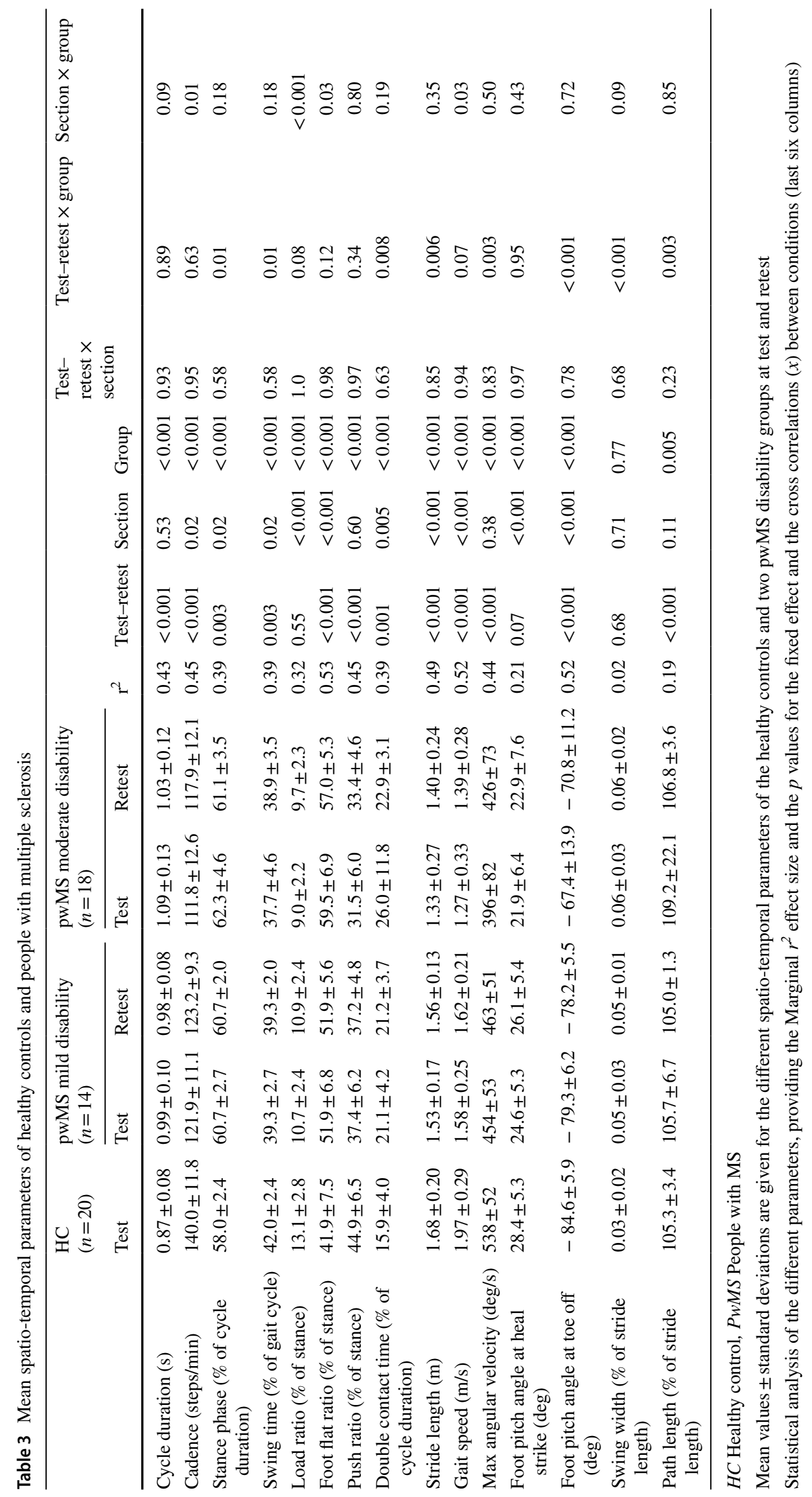



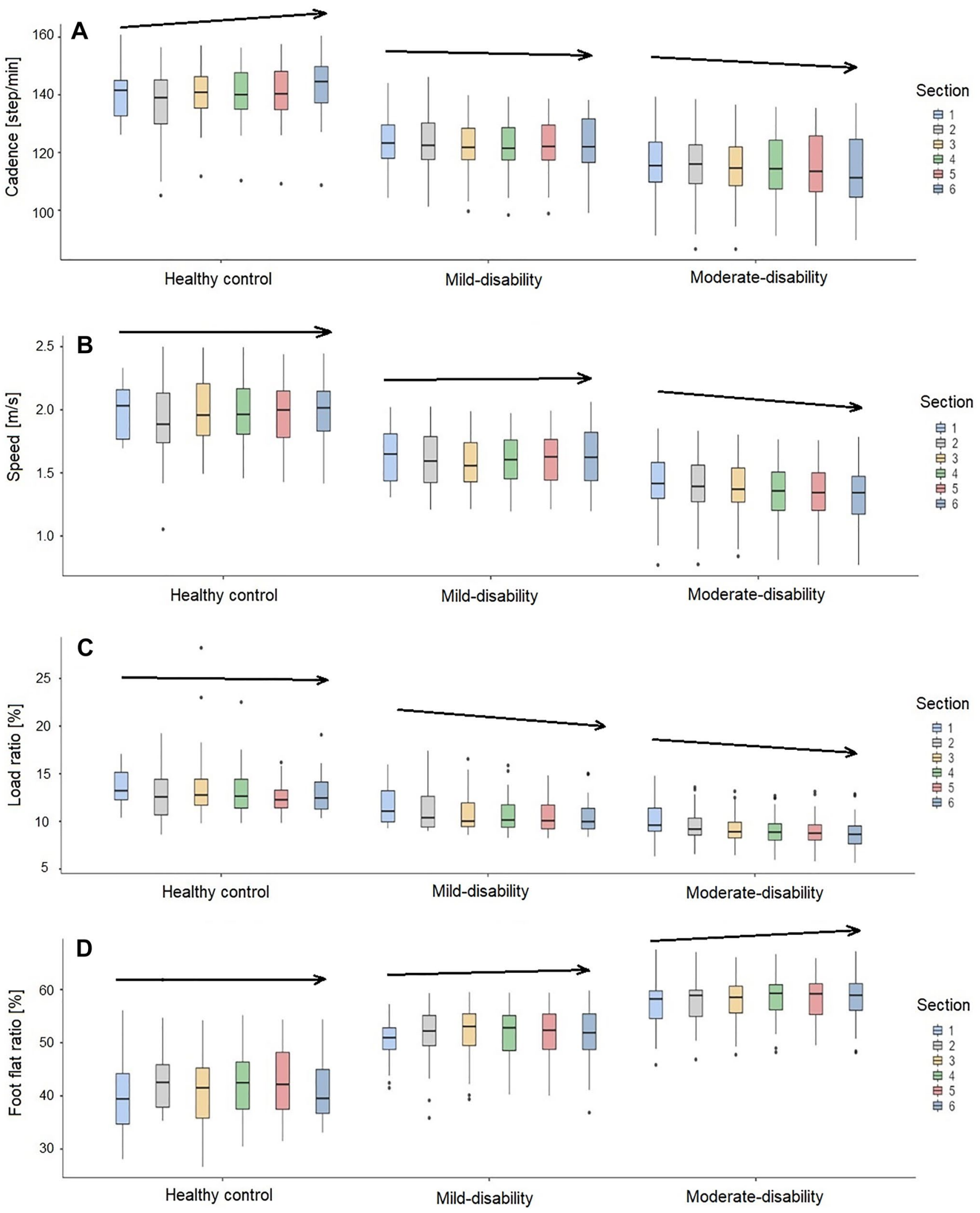

Fig. 2 Cross-correlation effect between the groups and the sections of the six-minute walk test. During the six intervals of 1 min each, the cadence (A), the speed (B), the load ratio $(\mathbf{C})$, and the foot flat ratio
(D) show a different behavior between the healthy controls, the milddisability, and the moderate-disability groups. Arrows indicate direction of change 

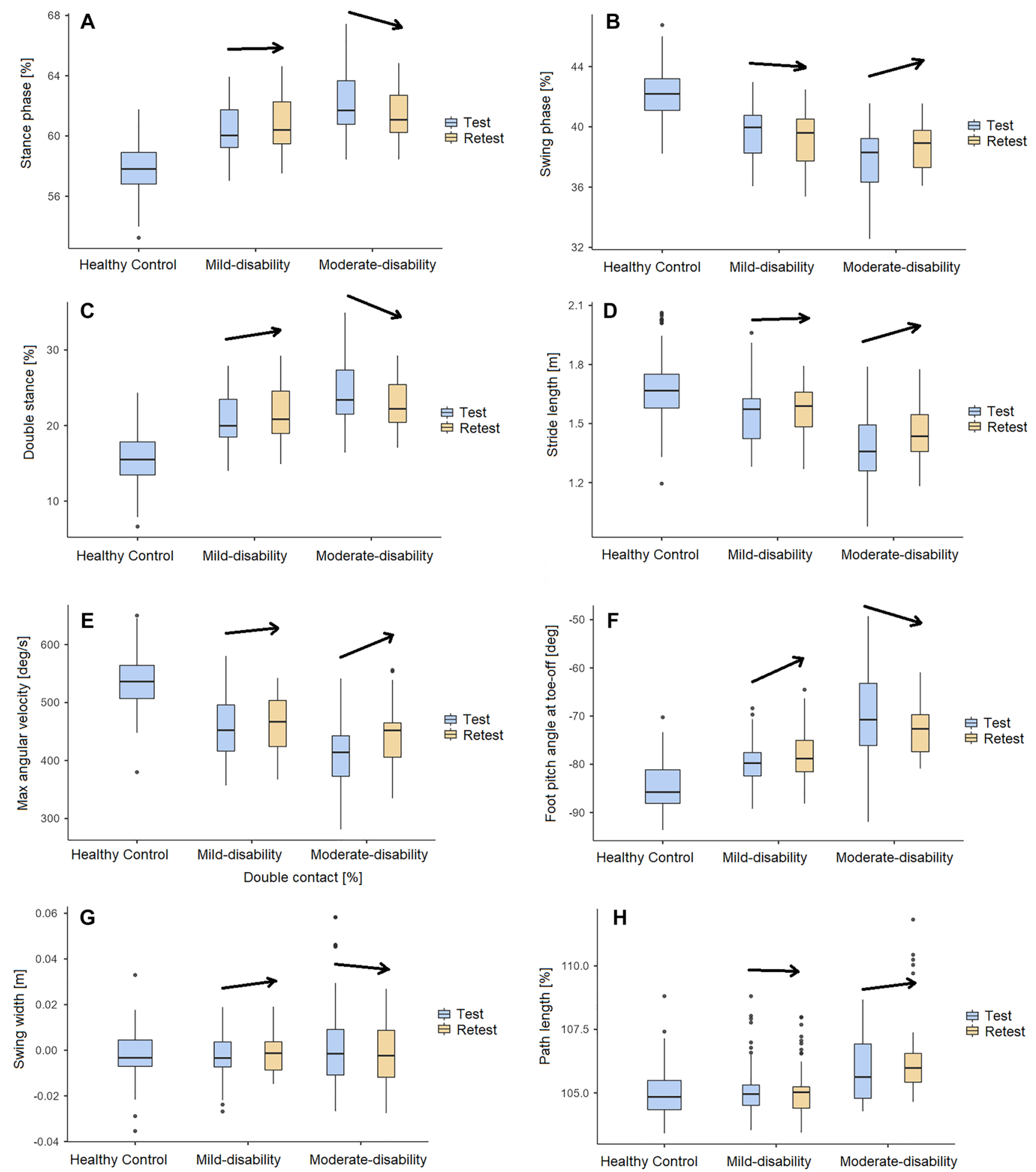

Fig. 3 Change in the selected gait parameters stance phase (A), swing phase $(\mathbf{B})$, double stance $(\mathbf{C})$, stride length $(\mathbf{D})$, max angular velocity $(\mathbf{E})$, toe-off pitch angle $(\mathbf{F})$, swing width $(\mathbf{G})$ and path length $(\mathbf{H})$

on the six-minute walk test, from baseline test to retest for the mildand moderate-disability pwMS groups. The HC group was tested only once. Arrows indicate direction of change

\section{Discussion}

standard testing with the 6MWT and as add-on to measure potential effects during a rehabilitation stay. The greater

This is the first study using IMUs in pwMS in relation to improvement in gait measures was found for the 


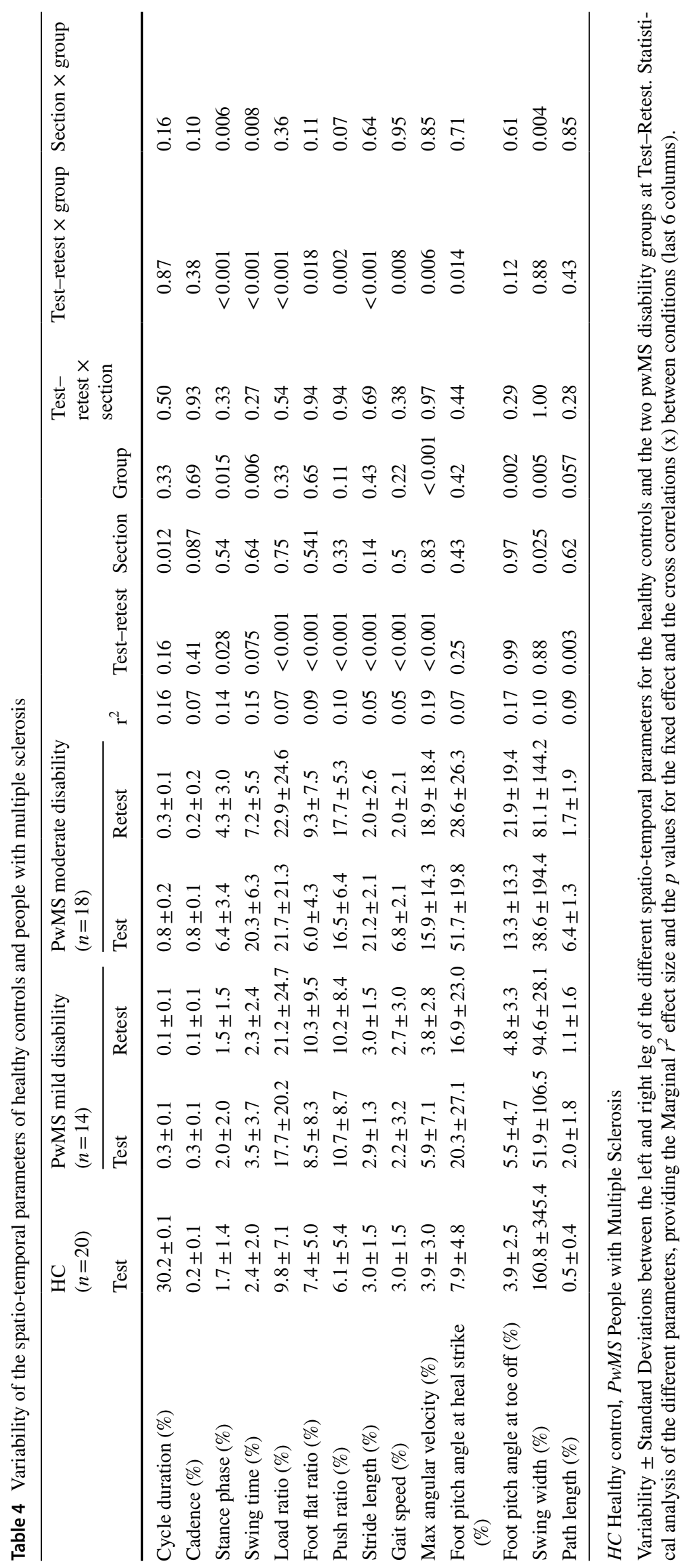


moderate-disability group. The gait parameter with the higher effect size, allowing the best differentiation between the disability groups, was the foot flat ratio $\left(R^{2}=0.53\right)$. Gait analyses from wearable sensors identified different evolutions of gait patterns during the 6MWT in pwMS with different physical disability. As expected, HC performed better than the mildly disabled pwMS, who again performed better than the moderately disabled pwMS on 6MWT. At the end of the stay, both disability groups walked longer on the 6MWT compared to baseline, but the overall change in the studied heterogeneous group was lower than the minimum $55 \mathrm{~m}$ or more than $20 \%$ improvement in walking distance defined as a minimal clinically important difference by others [33, 34]. However, for the moderate-disability group, it was higher than the clinically meaningful change of $21.6 \mathrm{~m}$ estimated for the 6MWT [6]. The immediate measured effect on gait with 6MWT during short-time rehabilitation stay was higher for pwMS with higher degree of disability which is in line with most studies on walking in MS finding the most pronounced efficacy in the more disabled groups [9]. One might speculate that bias toward quantitative data instead of combining qualitative and quantitative data, and measured and perceived (patient- and therapistreported) data might have influenced the existing data in the field. Moreover, analysis of data from the foot sensors identified significant differences in the gait patterns during the 6 min of the 6MWT between the HC group and the two pwMS groups. The evolution of the cadence, loading ratio, and foot flat ratio combined might be an interesting indicator of the walking ability. The moderate-disability group improved more than the mild-disability group from test to retest. Our findings are partly in line with a study of 58 pwMS with mild (EDSS $<4$ ) and moderate disability $(E D S S \geq 4)$ [35]. Shema-Shiratzky et al. found that specific gait features deteriorated over the course of the 6MWT, and were related to disability level and other clinical characteristics among pwMS. Subjects with moderate disability walked more poorly in most gait domains compared to the mild-disability group. As in our study, cadence was stable in the mildly disabled group, but decreased in the moderately disabled group during minute 1 and 6 . The authors found the same pattern for sample entropy but not for other automated gait measures. However, a control group was not included, and there was no retest or intervention. Angelini et al. [36] compared the performance of 6MWT in 57 progressive pwMS and $24 \mathrm{HC}$ by the use of body-worn sensors. Most of the 15 automated gait measures showed good-to-excellent between-session reliability. PwMS had longer step and stride durations/regularity, and had a less stable walk compared to controls. The abnormalities correlated with the level of disability and EDSS scores. The same group recently published an extension of this study [37]. A total of 114 pwMS were compared with $24 \mathrm{HC}$. Based on the sensor data from
6MWT, they developed a multifactorial model which was able to discriminate clinically relevant differences between pwMS with three disability levels.

A strength of the present study is the use of a standardized test protocol performed in a dedicated test room with stable conditions, without disturbances incorporated in a clinical rehabilitation stay setting. There were also only three, well-trained investigators, and we used a validated, commercially available sensor system with a dedicated software $\left(\mathrm{GaitUp}{ }^{\circledR}\right)$. There were also a relatively high number of pwMS and HC. A limitation of the study was that the HC were not tested twice, due to the restrictions introduced by the COVID-19 regulations. We could thus not rule out a possible learning effect from test to retest. However, the 6MWT is a relatively simple test with a low potential for learning, in contrast to other more complex tests, with reported good-toexcellent between-session reliability [36]. Also, a learning effect only in the more disabled group compared to the less disabled group is unlikely. Our study did not aim to compare different treatment strategies or evaluate therapeutic elements. There were differences in content, duration, and volume of rehabilitation in this real-world study with data retrieved during rehabilitation stays of 2-4 weeks. The MS center involved used their standard conventional rehabilitation approach with tailored multidiciplinary content for each participant. As a consequence, the participants were heterogeneous concerning type and number of interventions and goals. The common denominator was that the rehabilitation included a focus on physical rehabilitation related to mobility or walking. Some of the participants worked, e.g., specifically with cognitive behavioral and/or psychological approaches, included in their rehabilitation, so there are many potential factors of interest when searching for the active ingredients to improve walking capacity. Despite the heterogeneities, the positive results and trends are striking. If future studies aim to evaluate the specific effects of the interventions on, e.g., walking capacity or gait parameters, core elements of task-specific training, and defined quantitative and qualitative content and goals, adjusted to level of disability, should be included in the results.

There was similar age and BMI distribution in the $\mathrm{HC}$ and pwMS; however, there were more females in the HC group, which was adjusted for in the mixed model analysis. The mild-disability group had a higher BMI compared to both $\mathrm{HC}$ and the moderate-disability group, which might possibly have interfered with the results. Furthermore, the direction of the finding was in favor of the least disabled group, so that a possible confounder effect would have driven the results into even higher statistical differences. Fampridine may improve gait performance in up to $75 \%$ of pwMS [24], and introduction of such treatment during the rehabilitation stay could possibly also have interfered with the results. However, only one patient initiated and two discontinued 
such treatments during the stay, so fampridine is not considered a major contribution to the results. Three of the pwMS that were tested twice were using orthoses, and three were using canes. Patients using aids like orthoses is part of a real-life clinical patient setting, and we found it relevant to include these as well, though we are aware that this may have affected the gait measures. Unfortunately, the samples sizes were too small to control for this. However, by definition, all the patients that were in need of walking aids were in the moderate-disability group, which had the highest improvement. Since they were using the same device at test and retest, we think that the gait patterns should not have been significantly affected by this. The provided detailed quantitative information on gait pattern compared with the standard speed variable and distance-based walking tests aids tailored feedback and further intervention and monitoring strategies. Thus, the add-on detailed information aids the follow-up and can be investigated as a motivational facilitator, as well. The wearable sensors provide abundant information on gait patterns. Thus, it is crucial to pinpoint a few key spatiotemporal parameters which might be a practical and userfriendly approach. Also, the 6MWT may be burdensome for pwMS with higher degree of disability and be considered too time-consuming in busy clinical settings. Hence, proper adaption of wearable sensors with use of key parameters as a clinical practical application is of great importance, particularly for the potential as a fruitful clinical tool to grasp relevant quantitative aspects.

\section{Conclusions}

The use of wearable sensors as add-on in a conventional rehabilitation setting made it possible to include objective spatio-temporal parameters and qualitative walking assessments. The study identifies significant changes in interstride gait patterns which might be relevant qualitative gait changes to assess in a clinical setting. The gait parameter foot flat ratio, which allowed better differentiation between the disability groups, might be an interesting parameter in testing and follow-up to pick up change and differentiate concerning walking status. Gait analyses with wearable sensors during clinical testing identified different evolutions of gait patterns during the 6MWT in pwMS with different physical disability and HC. The immediate measured effect on gait with 6MWT during short-time rehabilitation stay was higher for pwMS with higher degree of disability. Standard walking tests do not necessarily measure change in walking capacity. The use of IMUs as add-on also allowed to identify significant changes in inter-stride gait patterns which might be relevant to identify when evaluating walking in pwMS. Thus, wearable sensors with proper adaptation and the use of key parameters have the potential to become useful clinical tools in evaluating and monitoring the disease in different clinical settings.

Supplementary Information The online version contains supplementary material available at https://doi.org/10.1007/s00415-022-10998-z.

Acknowledgements The present study is part of a large research project AutoActive: Tools and Methods for Autonomous Analysis of Human Activities from Wearable Device Sensor Data and unites a multidisciplinary research team with partners from MS Center Hakadal, Norwegian University of Science and Technology, Olympiatoppen, OUS, SINTEF, and University of Oslo. The project receives support from the Norwegian research council (project number 270791).

Funding Open access funding provided by University of Oslo (incl Oslo University Hospital).

\section{Declarations}

Conflicts of interest On behalf of all authors, the corresponding author states that there is no conflict of interest.

Ethical approval The protocol for the AutoActive project was approved by the local Data Protection Officer (DPO) at Oslo University Hospital (OUS) and MS Center Hakadal (MSSH). All participants provided written informed consent. The study was performed in accordance with the ethical standards laid down in the 1964 Declaration of Helsinki and its later amendments.

Open Access This article is licensed under a Creative Commons Attribution 4.0 International License, which permits use, sharing, adaptation, distribution and reproduction in any medium or format, as long as you give appropriate credit to the original author(s) and the source, provide a link to the Creative Commons licence, and indicate if changes were made. The images or other third party material in this article are included in the article's Creative Commons licence, unless indicated otherwise in a credit line to the material. If material is not included in the article's Creative Commons licence and your intended use is not permitted by statutory regulation or exceeds the permitted use, you will need to obtain permission directly from the copyright holder. To view a copy of this licence, visit http://creativecommons.org/licenses/by/4.0/.

\section{References}

1. Filippi M, Bar-Or A, Piehl F, Preziosa P, Solari A, Vukusic S, Rocca MA (2018) Multiple sclerosis. Nat Rev Dis Primers 4:43. https://doi.org/10.1038/s41572-018-0041-4

2. Zwibel HL (2009) Contribution of impaired mobility and general symptoms to the burden of multiple sclerosis. Adv Ther 26:10431057. https://doi.org/10.1007/s12325-009-0082-x

3. Kurtzke JF (1983) Rating neurologic impairment in multiple sclerosis: an expanded disability status scale (EDSS). Neurology 33:1444-1452. https://doi.org/10.1212/wnl.33.11.1444

4. Cohen JA, Reingold SC, Polman CH, Wolinsky JS, International Advisory Committee on Clinical Trials in Multiple S (2012) Disability outcome measures in multiple sclerosis clinical trials: current status and future prospects. Lancet Neurol 11:467-476. https://doi.org/10.1016/S1474-4422(12)70059-5

5. Goldman MD, Marrie RA, Cohen JA (2008) Evaluation of the sixminute walk in multiple sclerosis subjects and healthy controls. 
Mult Scler 14:383-390. https://doi.org/10.1177/1352458507 082607

6. Baert I, Freeman J, Smedal T, Dalgas U, Romberg A, Kalron A, Conyers H, Elorriaga I, Gebara B, Gumse J, Heric A, Jensen E, Jones K, Knuts K, Maertens de Noordhout B, Martic A, Normann B, Eijnde BO, Rasova K, Santoyo Medina C, Truyens V, Wens I, Feys P (2014) Responsiveness and clinically meaningful improvement, according to disability level, of five walking measures after rehabilitation in multiple sclerosis: a European multicenter study. Neurorehabil Neural Repair 28:621-631. https://doi.org/10.1177/ 1545968314521010

7. Shanahan CJ, Boonstra FMC, Cofre Lizama LE, Strik M, Moffat BA, Khan F, Kilpatrick TJ, van der Walt A, Galea MP, Kolbe SC (2017) Technologies for advanced gait and balance assessments in people with multiple sclerosis. Front Neurol 8:708. https://doi. org/10.3389/fneur.2017.00708

8. Brichetto G, Pedulla L, Podda J, Tacchino A (2019) Beyond center-based testing: understanding and improving functioning with wearable technology in MS. Mult Scler 25:1402-1411. https://doi.org/10.1177/1352458519857075

9. Leone C, Kalron A, Smedal T, Normann B, Wens I, Eijnde BO, Feys $P$ (2018) Effects of rehabilitation on gait pattern at usual and fast speeds depend on walking impairment level in multiple sclerosis. Int J MS Care 20:199-209. https://doi.org/10.7224/ 1537-2073.2015-078

10. Spain RI, Mancini M, Horak FB, Bourdette D (2014) Body-worn sensors capture variability, but not decline, of gait and balance measures in multiple sclerosis over 18 months. Gait Posture 39:958-964. https://doi.org/10.1016/j.gaitpost.2013.12.010

11. Chen S, Lach J, Lo B, Yang GZ (2016) Toward pervasive gait analysis with wearable sensors: a systematic review. IEEE J Biomed Health Inform 20:1521-1537. https://doi.org/10.1109/ JBHI.2016.2608720

12. Sasaki JE, Sandroff B, Bamman M, Motl RW (2017) Motion sensors in multiple sclerosis: narrative review and update of applications. Expert Rev Med Devices 14:891-900. https://doi.org/10. 1080/17434440.2017.1386550

13. Bradshaw MJ, Farrow S, Motl RW, Chitnis T (2017) Wearable biosensors to monitor disability in multiple sclerosis. Neurol Clin Pract 7:354-362. https://doi.org/10.1212/CPJ.0000000000000382

14. Atrsaei A, Dadashi F, Mariani B, Gonzenbach R, Aminian K (2021) Toward a remote assessment of walking bout and speed: application in patients with multiple sclerosis. IEEE J Biomed Health Inform 25:4217-4228. https://doi.org/10.1109/jbhi.2021. 3076707

15. Trojaniello D, Cereatti A, Pelosin E, Avanzino L, Mirelman A, Hausdorff JM, Della Croce U (2014) Estimation of step-by-step spatio-temporal parameters of normal and impaired gait using shank-mounted magneto-inertial sensors: application to elderly, hemiparetic, parkinsonian and choreic gait. J Neuroeng Rehabil 11:152. https://doi.org/10.1186/1743-0003-11-152

16. Mariani B, Hoskovec C, Rochat S, Büla C, Penders J, Aminian $\mathrm{K}$ (2010) 3D gait assessment in young and elderly subjects using foot-worn inertial sensors. J Biomech 43:2999-3006. https://doi. org/10.1016/j.jbiomech.2010.07.003

17. Johansson D, Malmgren K, Alt Murphy M (2018) Wearable sensors for clinical applications in epilepsy, Parkinson's disease, and stroke: a mixed-methods systematic review. J Neurol 265:17401752. https://doi.org/10.1007/s00415-018-8786-y

18. Comber L, Galvin R, Coote S (2017) Gait deficits in people with multiple sclerosis: a systematic review and meta-analysis. Gait Posture 51:25-35. https://doi.org/10.1016/j.gaitpost.2016.09.026

19. Socie MJ, Motl RW, Pula JH, Sandroff BM, Sosnoff JJ (2013) Gait variability and disability in multiple sclerosis. Gait Posture 38:51-55. https://doi.org/10.1016/j.gaitpost.2012.10.012
20. Martin CL, Phillips BA, Kilpatrick TJ, Butzkueven H, Tubridy N, McDonald E, Galea MP (2006) Gait and balance impairment in early multiple sclerosis in the absence of clinical disability. Mult Scler 12:620-628. https://doi.org/10.1177/1352458506070658

21. Sosnoff JJ, Sandroff BM, Motl RW (2012) Quantifying gait abnormalities in persons with multiple sclerosis with minimal disability. Gait Posture 36:154-156. https://doi.org/10.1016/j.gaitpost.2011. 11.027

22. Storm FA, Cesareo A, Reni G, Biffi E (2020) Wearable inertial sensors to assess gait during the 6-minute walk test: a systematic review. Sensors (Basel). https://doi.org/10.3390/s20092660

23. Baert I, Smedal T, Kalron A, Rasova K, Heric-Mansrud A, Ehling R, Elorriaga Minguez I, Nedeljkovic U, Tacchino A, Hellinckx P, Adriaenssens G, Stachowiak G, Gusowski K, Cattaneo D, Borgers S, Hebert J, Dalgas U, Feys P (2018) Responsiveness and meaningful improvement of mobility measures following MS rehabilitation. Neurology 91:e1880-e1892. https://doi.org/10.1212/WNL. 0000000000006532

24. Allart E, Benoit A, Blanchard-Dauphin A, Tiffreau V, Thevenon A, Zephir H, Outteryck O, Lacour A, Vermersch P (2015) Sustained-released fampridine in multiple sclerosis: effects on gait parameters, arm function, fatigue, and quality of life. J Neurol 262:1936-1945. https://doi.org/10.1007/s00415-015-7797-1

25. ATS Committee on Proficiency Standards for Clinical Pulmonary Function Laboratories (2002) ATS statement: guidelines for the six-minute walk test. Am J Respir Crit Care Med 166:111-117. https://doi.org/10.1164/ajrccm.166.1.at1102

26. Podsiadlo D, Richardson S (1991) The timed "Up \& Go": a test of basic functional mobility for frail elderly persons. J Am Geriatr Soc 39:142-148. https://doi.org/10.1111/j.1532-5415.1991.tb016 16. $\mathrm{x}$

27. Crosbie WJ, Nimmo MA, Banks MA, Brownlee MG, Meldrum F (1989) Standing balance responses in two populations of elderly women: a pilot study. Arch Phys Med Rehabil 70:751-754

28. Fischer JS, Rudick RA, Cutter GR, Reingold SC (1999) The Multiple Sclerosis Functional Composite Measure (MSFC): an integrated approach to MS clinical outcome assessment. National MS Society Clinical Outcomes Assessment Task Force. Mult Scler 5:244-250. https://doi.org/10.1177/135245859900500409

29. Nieuwenhuis MM, Van Tongeren H, Sorensen PS, Ravnborg M (2006) The six spot step test: a new measurement for walking ability in multiple sclerosis. Mult Scler 12:495-500. https://doi. org/10.1191/1352458506ms1293oa

30. Mariani B, Rouhani H, Crevoisier X, Aminian K (2013) Quantitative estimation of foot-flat and stance phase of gait using footworn inertial sensors. Gait Posture 37:229-234. https://doi.org/ 10.1016/j.gaitpost.2012.07.012

31. Mariani B, Jiménez MC, Vingerhoets FJ, Aminian K (2013) Onshoe wearable sensors for gait and turning assessment of patients with Parkinson's disease. IEEE Trans Biomed Eng 60:155-158. https://doi.org/10.1109/tbme.2012.2227317

32. Cohen J (1992) A power primer. Psychol Bull 112:155-159. https://doi.org/10.1037//0033-2909.112.1.155

33. Applebee A, Goodman AD, Mayadev AS, Bethoux F, Goldman MD, Klingler M, Blight AR, Carrazana EJ (2015) Effects of dalfampridine extended-release tablets on 6-minute walk distance in patients with multiple sclerosis: a post hoc analysis of a doubleblind, placebo-controlled trial. Clin Ther 37:2780-2787. https:// doi.org/10.1016/j.clinthera.2015.10.014

34. Sola-Valls N, Blanco Y, Sepulveda M, Llufriu S, MartinezLapiscina EH, Zubizarreta I, Pulido-Valdeolivas I, Montejo C, Villoslada P, Saiz A (2018) Combined walking outcome measures identify clinically meaningful response to prolonged-release fampridine. Ther Adv Neurol Disord 11:1756286418780007. https:// doi.org/10.1177/1756286418780007 
35. Shema-Shiratzky S, Gazit E, Sun R, Regev K, Karni A, Sosnoff JJ, Herman T, Mirelman A, Hausdorff JM (2019) Deterioration of specific aspects of gait during the instrumented 6-min walk test among people with multiple sclerosis. J Neurol 266:3022-3030. https://doi.org/10.1007/s00415-019-09500-z

36. Angelini L, Hodgkinson W, Smith C, Dodd JM, Sharrack B, Mazza C, Paling D (2020) Wearable sensors can reliably quantify gait alterations associated with disability in people with progressive multiple sclerosis in a clinical setting. J Neurol 267:2897-2909. https://doi.org/10.1007/s00415-020-09928-8

37. Angelini L, Buckley E, Bonci T, Radford A, Sharrack B, Paling D, Nair KPS, Mazza C (2021) A multifactorial model of multiple sclerosis gait and its changes across different disability levels. IEEE Trans Biomed Eng. https://doi.org/10.1109/TBME.2021. 3061998 